\title{
Inflation with light Weyl ghost
}

\author{
Anna Tokareva ${ }^{1, \star}$ \\ ${ }^{1}$ INR RAS, 60-letiya Oktyabrya prospekt 7a, Moscow 117312, Russia
}

\begin{abstract}
Inflationary perturbations are considered in a renormalizable but non-unitary theory of gravity with the additional Weyl term. We obtained that ghost degrees of freedom do not spoil the inflation and the scalar perturbation amplitude at the linear level even in a case of the ghost with mass smaller than Hubble parameter at inflation. The ghost impact to the observables is also estimated to be negligible for the range of masses allowed by the experiment. The non-linear level of the theory and its possible application are also discussed.
\end{abstract}

\section{Introduction}

General relativity, providing a good explanation for all known experimental results, fails all attempts to quantize it consistently. The Einstein-Hilbert action leads to the non-renormalizable theory which means the loss of its predictive force at Planck energies. Despite the low energy physics is described by the effective field theory, there are examples showing that some processes below the Planck scale (such as inflation and reheating) may be sensitive to the UV completion of the theory $[1,2]$.

It is well known that there is a way to construct the renormalizable theory of gravity by the price of unitarity. The quadratic in curvature action for gravity was studied by Stelle in Refs. [3, 4]. This action,

$$
S=\frac{M_{P}^{2}}{2} \int d^{4} x \sqrt{-g}\left(R-\frac{1}{2 m^{2}} C_{\mu \nu \rho \sigma} C^{\mu \nu \rho \sigma}+\frac{1}{6 m_{0}^{2}} R^{2}\right)
$$

when linearised on the flat space background, provides usual massless graviton, scalar with mass $m_{0}$ and spin-2 ghost degree of freedom with mass $m$. Here $C_{\mu \nu \rho \sigma}$ stands for the Weyl tensor and $M_{P}$ is the reduced Planck mass. The adimentional variant of this action was recently discussed in [5].

Despite the interpretation of non-unitary theories is a complicated problem such theories might be useful in the sense of an effective description. In particular, the non-interacting ghost is not dangerous because it may be consistently quantized with the negative norm and positive energy (see for example Ref. [6] for the discussion). In this paper, we discuss the limit in which the Weyl ghost becomes interacting very weakly and hereafter we impose the negative norm prescription for the ghost states. Since it has only derivative coupling to matter we expect that this limit corresponds to the light ghost. In the next section we estimate the typical time of ghost amplification and find out the allowed range for the ghost mass to be $m \lesssim 10 \mathrm{MeV}$ providing the lifetime of ghost instability to be much larger than the Universe age.

^e-mail: tokareva@ms2.inr.ac.ru 
In order to check the validity of the effective description in terms of non-unitary theory we calculate the evolution of the scalar perturbations in a theory with light Weyl ghost starting from vacuum initial conditions (this case is not yet studied in literature). We obtained the deviations from the standard cosmology to be negligible for the discussing range of masses. The ghost impact to the tensor perturbations has been found in a paper [7]. It looks interesting that the amplitude of tensor perturbations (which is observable in cosmology) in a case of light ghost is damped by a factor $m / \sqrt{2} H_{0}$, where $H_{0}$ stands for the Hubble parameter at the inflationary stage.

The paper is organised in the following way. In the Section 2 we show that the limit of light ghost really corresponds to low rates of ghost creation and decay. In the Section 3 we calculate the scalar perturbations on the inflationary stage in a gravity with the Weyl term for the case of the light ghost. In the Section 4 we follow the evolution of scalar perturbations up to the late Universe and obtain the ghost impact on CMB. In the last section, we come to conclusions.

\section{The discussion about the light ghost}

In a paper [5] the naturalness problem for the Higgs mass in a case of the heavy ghost was pointed out. This problem may be avoided when the ghost is lighter than $10^{10} \mathrm{GeV}$ which is less than the usual Hubble parameter at inflation. Preserving the Higgs mass naturalness motivates the study of the inflationary perturbations in a case of the ghost being lighter than the Hubble parameter. We must require for the first that the instability connected with the ghost decay (which is actually the ghost number amplification because of the negative width) evolves very slowly, corresponding to the ghost decay width much larger than the Universe lifetime. With negative norm prescription the ghost decay width may be estimated as $[8,9]$

$$
\Gamma \sim-\frac{m^{3}}{M_{P}^{2}}
$$

In order to have a ghost lifetime to be much larger than the age of Universe we must have $m \ll 10$ $\mathrm{MeV}$.

Another process with the negative probability which must be negligible in the considering limit of the light ghost is the ghost production in a hot plasma. Although there is a problem of interpretation with the negative cross sections we take as the estimation of the ghost effects values calculated in a usual way. We approximate the cross section as

$$
\sigma \sim-\frac{e^{2}}{M_{P}^{2}} .
$$

Here $e$ stands for the electron charge. We obtain the number of produced ghosts which corresponds to their impact on the energy density of Universe being equal to

$$
\Omega_{g h}=0.02 \frac{m}{10 \mathrm{MeV}} \frac{T_{\max }}{10^{13} \mathrm{GeV}} .
$$

Here $T_{\max }$ is the maximal temperature that has ever been in the Universe. One can see again that for the ghost lighter than $10 \mathrm{MeV}$ and natural values of the reheating temperature a number of ghosts turn out to be negligible.

Ghosts with too low masses cannot be considered because of experimental constraints for the gravity at short distances. The limit coming from recent measurements [10] is $m>10^{-2} \mathrm{eV}$ which leaves a wide range ( 9 orders of magnitude) for the weakly coupled ghost we discuss in this paper. 


\section{Scalar perturbations at the inflationary stage}

\subsection{Common equations}

We consider the action

$$
S=\frac{M_{P}^{2}}{2} \int d^{4} x \sqrt{-g} R-\frac{1}{2} \int d^{4} x \sqrt{-g}\left(\partial_{\mu} \phi \partial^{\mu} \phi+2 V(\phi)\right)-\frac{M_{P}^{2}}{4 m^{2}} \int d^{4} x \sqrt{-g} C_{\mu \nu \rho \sigma} C^{\mu \nu \rho \sigma},
$$

where $g$ is the determinant of the metric $g_{\mu \nu}, R$ is the scalar curvature and $C_{\mu \nu \rho \sigma}$ is the Weyl tensor. ${ }^{1}$

This action describes the usual Einstein gravity corrected by the Weyl curvature term. The scalar $\phi$ with the proper potential may be appropriate for inflation. If we consider the most general quadratic in curvature corrections to the Einstein gravity action than the $R^{2}$ term may be rewritten in the Einstein frame through the additional scalaron field $\phi$ [11]. So the action (5) with a special form of $V(\phi)$ may describe the general second order corrected gravity which with the specially tuned scalaron mass may provide the phenomenologically viable Starobinsky inflation [12].

We start to work in the newtonian gauge [13] where the metric and inflaton perturbations take the form:

$$
d s^{2}=a(\eta)^{2}\left\{-(1+2 \Psi) d \eta^{2}+2 \bar{\Psi}_{i} d \eta d x^{i}+\left[(1+2 \Phi) \delta_{i j}+\bar{h}_{i j}\right] d x^{i} d x^{j}\right\}, \quad \delta \phi=\chi .
$$

Here $\bar{h}_{i j}$ correspond to the tensor modes, $\bar{\Psi}_{i}$ - to the vector modes and the scalar sector is described by $\Phi, \Psi$ and $\chi$.

The expansion of the action (5) at quadratic order in the perturbations is presented in Ref. [14]:

$$
S^{(\mathrm{S})}=S_{\mathrm{E}}^{(\mathrm{S})}-\frac{M_{P}^{2}}{3 m^{2}} \int d^{4} x[\Delta(\Psi-\Phi)]^{2},
$$

where

$$
\begin{gathered}
S_{\mathrm{E}}^{(\mathrm{S})}=\frac{M_{P}^{2}}{2} \int d^{4} x a^{2}\left[-6 \Phi^{\prime 2}+12 \mathcal{H} \Psi \Phi^{\prime}+2 \partial_{i} \Phi\left(2 \partial^{i} \Psi+\partial^{i} \Phi\right)-2\left(\mathcal{H}^{\prime}+2 \mathcal{H}^{2}\right) \Psi^{2}\right. \\
\left.+\frac{1}{M_{P}^{2}}\left(\chi^{\prime 2}-\partial_{i} \chi \partial^{i} \chi-a^{2} V_{, \phi \phi} \chi^{2}-6 \phi^{\prime} \Phi^{\prime} \chi-2 \phi^{\prime} \chi^{\prime} \Psi-2 a^{2} V_{, \phi} \Psi \chi\right)\right] .
\end{gathered}
$$

Here the primes mean the derivatives with respect to conformal time $\eta$, and $\mathcal{H}=a^{\prime} / a$ is the conformal Hubble parameter. The action describes two coupled dynamical fields: a ghost and an inflaton while $\Psi$ is to be integrated out.

\subsection{Initial conditions and solutions for the de Sitter space}

In a limit of de Sitter space the action may be diagonalized. Here we use $a(\eta)=-1 /\left(H_{0} \eta\right)$ where $H_{0}$ is the Hubble parameter at inflation. The action for the inflaton perturbation takes the usual form:

$$
S_{\chi}=\frac{1}{2} \int d^{4} x \frac{1}{H_{0}^{2} \eta^{2}}\left(\chi^{\prime 2}-\left(\partial_{i} \chi\right)^{2}\right),
$$

\footnotetext{
${ }^{1}$ Units: $M_{P}=1 / \sqrt{8 \pi G}, c=1$. Conventions: $(-+++) ; R_{\nu \rho \sigma}^{\mu}=\partial_{\rho} \Gamma_{v \sigma}^{\mu}-\partial_{\sigma} \Gamma_{v \rho}^{\mu}+\cdots ; R_{v \sigma}=R^{\mu}{ }_{v \mu \sigma} ; R=g^{\mu \nu} R_{\mu \nu}$; $G_{\mu \nu}=R_{\mu \nu}-\frac{1}{2} g_{\mu \nu} R . C_{\mu \nu \rho \sigma}=R_{\mu \nu \rho \sigma}-\frac{1}{2}\left(g_{\mu \rho} G_{v \sigma}-g_{\mu \sigma} G_{\nu \rho}-g_{v \rho} G_{\mu \sigma}+g_{v \sigma} G_{\mu \rho}\right)-\frac{R}{3}\left(g_{\mu \rho} g_{v \sigma}-g_{\mu \sigma} g_{v \rho}\right)$. Greek indices run from 0 to 3 ; latin indices run from 1 to 3 .
} 
After integrating out $\Psi$ one obtain the action for the ghost perturbation:

$$
S_{\Phi}=\int d \eta d^{3} q \frac{3 M_{P}^{2}}{H_{0}^{2}}\left[-\frac{\beta q^{4} \eta^{2}}{9+\beta q^{4} \eta^{4}} \Phi^{\prime}(q) \Phi^{\prime}(-q)+\frac{3 q^{4}\left(\beta^{2} q^{6} \eta^{6}+\beta q^{4} \eta^{4}(1-4 \beta)-3 \beta q^{2} \eta^{2}+9\right)}{\left(9+\beta q^{4} \eta^{4}\right)^{2}} \Phi(q) \Phi(-q)\right]
$$

Here $\beta=H_{0}^{2} / m^{2}$. The vacuum initial conditions for the inflaton and ghost perturbations are to be set at $\eta \rightarrow-\infty$ in the subhorizon limit. The action for the inflaton behaves as in the usual gravity while the action for the ghost becomes

$$
S_{\Phi}=\int d^{4} x \frac{3 M_{P}^{2}}{H_{0}^{2} \eta^{2}}\left[-\Phi^{\prime 2}+\left(\partial_{i} \Phi\right)^{2}\right] .
$$

After canonical normalization one can obtain that the vacuum initial conditions lead to the field perturbations at $\eta \rightarrow-\infty$ :

$$
\Phi=\frac{H_{0} q \eta}{2 \pi \sqrt{6} M_{P}} e^{-i q \eta}, \quad \chi=\frac{H_{0} q \eta}{2 \pi} e^{-i q \eta}
$$

In the opposite limit $\eta \rightarrow 0$ one obtain an equation

$$
\Phi^{\prime \prime}+\frac{2}{\eta} \Phi^{\prime}+\frac{1}{\beta \eta^{2}} \Phi=0
$$

while the equation for $\chi$ is standard [13]. The solution for ghost consists of the modes: $\Phi \propto \eta^{-1}$ and $\Phi \propto \eta^{-1 / \beta} \approx$ const. We see that there is a growing as a scale factor mode in the set of solutions for the ghost field. The initial conditions lead for this mode to be normalised as (up to a factor of order unity)

$$
\Phi=\frac{H_{0}}{2 \pi \sqrt{6} M_{P}} \frac{a H_{0}}{q} .
$$

Later we will see that this instability is not physical, it is connected with the choice of gauge.

\subsection{Slow roll inflation}

The real inflationary stage lead to small deviations from the exact de Sitter space so the equations on $\Phi$ and $\chi$ following from the action (7) becomes coupled. In the superhorizon limit and within the slow roll approximation the equation for $\chi$ is $^{2}$

$$
\Psi=\frac{3 M_{P}^{2} H}{V} \dot{\Phi}, \ddot{\chi}+3 H \dot{\chi}=-\dot{\phi}(3 \dot{\Phi}-\dot{\Psi})-2 V_{, \phi} \Psi .
$$

Since $\Phi$ has a growing mode (14) it induce the growing solution for $\chi$ through the upper equation. In the slow roll approximation $V=3 H^{2} M_{P}^{2}, V_{, \phi}=-3 H \dot{\phi}$ so for $\chi$ one obtain

$$
\ddot{\chi}+3 H \dot{\chi}=4 H \dot{\phi} \frac{H_{0}^{2}}{2 \pi \sqrt{6} M_{P}} \frac{a}{q},
$$

which provides the solution (up to the falling sub-dominate modes):

$$
\chi=\frac{H_{0}}{2 \pi}+\frac{\dot{\phi}}{H} \frac{H_{0}^{2}}{2 \pi \sqrt{6} M_{P}} \frac{a}{q} .
$$

In order to show that the growing modes are artefacts of the choice of gauge in the next section we calculate the metric perturbations in the comoving gauge.

\footnotetext{
${ }^{2}$ Hereafter we work in the physical time $t, H=\dot{a} / a$ is the usual Hubble parameter
} 


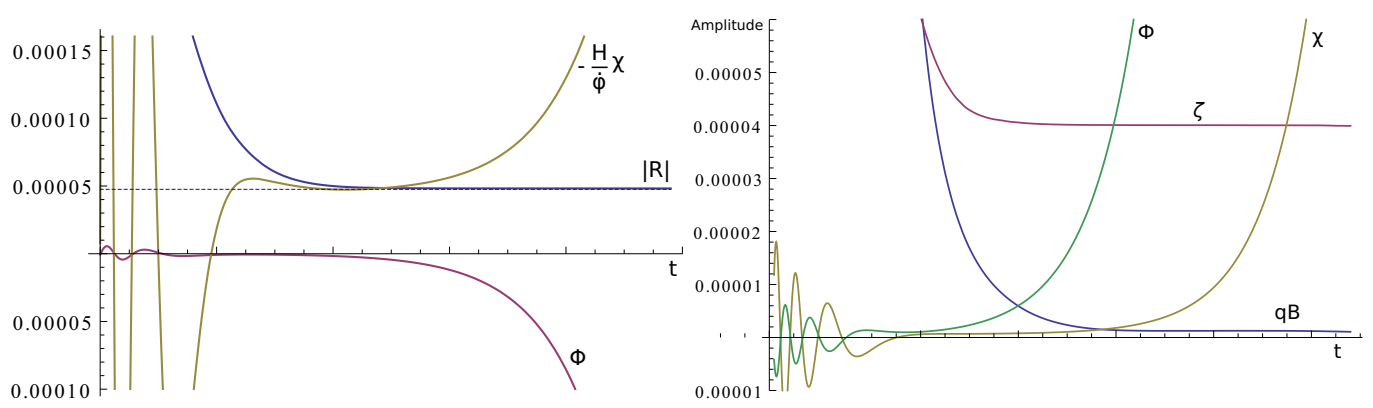

Figure 1. Left plot shows the cancellation between the growing modes of $\Phi$ and $\chi$ when the metric perturbation in a comoving gauge $\zeta$ is calculated. Right plot illustrates the time evolution for the discussed perturbation amplitudes in Newtonian and comoving gauges.

\subsection{The perturbations in the comoving gauge}

The comoving gauge is defined by the condition $\delta \phi=0$. The metric takes the form

$$
g_{00}=a^{2}(1+2 A), g_{0 i}=2 a^{2} \partial_{i} B, g_{i j}=a^{2}\left(\delta_{i j}(1+2 \zeta)+\partial_{i} \partial_{j} E\right)
$$

Here only scalar part of perturbations is considered. The perturbation $E$ may be set to be zero by the special choice for one of the two gauge transformation parameters. The other parameter fixes the condition $\delta \phi=0$ [13]. The parameter $A$ is falling approximately as $1 / a$ in the superhorizon limit so we concentrate on the perturbations $\zeta$ and $B$ connected with variables from Newtonian gauge as

$$
\zeta=\Phi-\frac{H}{\dot{\phi}} \chi, \quad B=\frac{\chi}{a \dot{\phi}}
$$

Substituting our solutions (14) and (17) we obtain

$$
\zeta=-\frac{H_{0}^{2}}{2 \pi \dot{\phi}}=\frac{H_{0}}{2 \pi \sqrt{2 \epsilon} M_{P}}, \quad B=\frac{H_{0}}{2 \pi \sqrt{6} q M_{P}}=-\sqrt{\frac{\epsilon}{3}} \frac{\zeta}{q} .
$$

Here $\epsilon=M_{P}^{2}\left(V_{, \phi} / V\right)^{2} / 2$ is the first slow roll parameter. We see that growing modes in $\Phi$ and $\chi$ cancel each other providing the value of $\zeta$ to be the same as in the usual Einstein gravity defining the amplitude of curvature perturbation that is known to be $\zeta=10^{-5}$. The difference is in the component $B$ : in usual gravity it is falling as $1 / a$ while in the considered model it is constant. The quantity which actually stands in metric, $\partial_{i} B=n_{i} \sqrt{\epsilon / 3} \zeta \ll 1$ (where $n_{i}$ ia a unit vector) is much smaller than unity proving that the linear regime is consistent in the comoving gauge being broken in the Newtonian gauge.

All the discussed solutions are plotted in a Figure 1. In order to obtain the possible phenomenological consequences of non-vanishing perturbation $B$ we need to follow its evolution after inflation up to the late Universe. We will do it in the next section.

\section{Evolution of cosmological perturbations after inflation}

\subsection{Equations}

The comoving gauge is convenient for understanding of the superhorizon evolution due to the good behaviour of the metric components. But it is much more easy to solve the equations on the variables 
defined in the Newtonian gauge. The combination $W=\Psi-\Phi$ has a very important property: it is possible to write all Einstein equations as one fourth order equation on $W$. After solving it one can easily obtain all the perturbations in the comoving gauge using formulas given in [14]. For the inflationary stage (or stage dominated by the scalar field) the master equation has been derived in [14]:

$$
\frac{1}{m^{2}}\left(W^{(4)}-\frac{\ddot{H}}{\dot{H}} W^{(3)}\right)+C_{2} \ddot{W}+C_{1} \dot{W}+C_{0} W=0,
$$

where

$$
\left\{\begin{array}{l}
C_{2}=1+\frac{1}{m^{2}}\left(\frac{2 q^{2}}{a^{2}}-H^{2}\right) \\
C_{1}=H-\frac{\ddot{H}}{\dot{H}}+\frac{1}{m^{2}}\left[\frac{H^{2} \ddot{H}}{\dot{H}}-3 H \dot{H}-\frac{q^{2}}{a^{2}}\left(\frac{\ddot{H}}{\dot{H}}+4 H\right)\right] \\
C_{0}=2 \dot{H}-\frac{H \ddot{H}}{\dot{H}}+\frac{q^{2}}{a^{2}}+\frac{q^{2}}{m^{2} a^{2}}\left(\frac{H \ddot{H}}{\dot{H}}-\frac{4}{3} \dot{H}+2 H^{2}+\frac{q^{2}}{a^{2}}\right)
\end{array} .\right.
$$

This equation was derived for the stage dominated by the scalar field. To proceed with the hot stage we need to obtain a similar equation from the set of Einstein equations. At the stages dominated by the fluid (matter or radiation) they take the form:

$$
\begin{gathered}
\frac{q^{2}}{a^{2}} \Phi+3 H(\dot{\Phi}-H \Psi)-\frac{1}{3 m^{2}} \frac{q^{4}}{a^{4}} W=\frac{\delta \rho}{2 M_{p}^{2}}, \\
-\ddot{\Phi}-3 H \dot{\Phi}+H \dot{\Psi}+\Psi\left(2 \dot{H}+3 H^{2}\right)-\frac{q^{2}}{3 a^{2}}(\Psi+\Phi)-\frac{1}{9 m^{2}} \frac{q^{4}}{a^{4}} W=\frac{\delta p}{2 M_{p}^{2}}, \\
-(\Phi+\Psi)=\frac{1}{m^{2}}\left(\ddot{W}+H \dot{W}+\frac{q^{2}}{3 a^{2}} W\right) .
\end{gathered}
$$

The first equation is (00) component of Einstein equations and the other two are the trace and traceless part of $(i j)$ component. The $(0 i)$ component may be considered as the definition of velocity perturbation. For adiabatic perturbations the equation of state connects $\delta p$ and $\delta \rho$ as

$$
\delta p=c_{s}^{2} \delta \rho, \quad c_{s}^{2}=-1-\frac{\ddot{H}}{3 H \dot{H}}
$$

which allows us to obtain the closed equation on $W$ like in the case of inflation driven by scalar field.

$$
\frac{1}{m^{2}}\left(W^{(4)}-\frac{\ddot{H}}{\dot{H}} W^{(3)}\right)+A_{2} \ddot{W}+A_{1} \dot{W}+A_{0} W=0,
$$

where

$$
\left\{\begin{array}{l}
A_{2}=1+\frac{1}{m^{2}}\left(\frac{q^{2}}{3 a^{2}} \frac{\ddot{H}}{H \dot{H}}-H^{2}\right) \\
A_{1}=H-\frac{\ddot{H}}{\dot{H}}+\frac{1}{m^{2}}\left[\frac{H^{2} \ddot{H}}{\dot{H}}-3 H \dot{H}-\frac{q^{2}}{a^{2}}\left(\frac{2 \ddot{H}}{3 \dot{H}}+2 H\right)\right] \\
A_{0}=2 \dot{H}-\frac{H \ddot{H}}{\dot{H}}-\frac{q^{2}}{a^{2}}\left(\frac{\ddot{H}}{3 H \dot{H}}\right)+\frac{q^{2}}{m^{2} a^{2}}\left(\frac{H \ddot{H}}{\dot{H}}-4 \dot{H}+2 H^{2}\right)-\frac{q^{4}}{m^{2} a^{4}}\left(1+\frac{\ddot{H}}{3 H \dot{H}}\right) .
\end{array}\right.
$$

One can see that equation (27) differs from (21) only by terms proportional to $q^{2}$ so in the superhorizon regime both equations coincide. All modes that we can observe are in the superhorizon regime at the moment of transition from inflation to the matter stage so we do not care about the difference in equations for the subhorizon modes. 


\subsection{Solutions in the superhorizon regime}

Now we consider the common solution of equation (21) (or (27) since they coincide in the superhorizon regime). Until $H \gg m$ holds this equation may be analytically solved. The four solutions are:

$$
W_{c}, \int a d t, \int \frac{d t}{a}, \int a d t_{1} \int^{t_{1}} \frac{H}{a^{2}} d t_{2} \int^{t_{2}} \frac{a \dot{H}}{H^{2}} d t_{3}
$$

The first mode $W_{c}$ correspond to $\zeta=$ const and this solution provide the main impact to $\zeta$ in the limits $q / a \ll H$ and $H \gg m$. In the Section 3.4 it was shown that at inflation $\zeta$ behaves exactly as in the usual gravity. The exact conservation of $\zeta$ in the considering model was proved in [14]. $W_{c}$ is connected to $\zeta$ as:

$$
W_{c}=-2 \epsilon \zeta-\text { inflation, } W_{c}=-\frac{2 \zeta}{p+1}-\text { stage } a \propto t^{p} .
$$

The second mode growing as $\int a d t$ provides the main constant impact to the comoving perturbation $B$ which is absent in Einstein gravity. One can see that $B$ is conserved until $H \gg m$ holds independently of the stage of the Universe expansion.

The last solution at inflationary stage grows as $t$ while at matter stage it behaves as $t^{2}$. This solution may be combined with $W_{c}$ in such a way that it would give the only subdominant (and decreasing) impact to $\zeta$ and $B$.

When the Hubble parameter becomes smaller then the ghost mass, $H \ll m$, the ghost modes start oscillate even in the superhorizon regime. The solutions for $W$ in that case were obtained in [14] for the stage $a \propto t^{p}$ :

$$
W_{c}, t^{-p-1}, t^{p / 2} e^{ \pm i m t} .
$$

The first two modes correspond to the Einstein modes and the growing oscillations are special solutions for Weyl gravity. As it was noted in [14] the growing modes do not give an impact to $\zeta$. Again only mode $W_{c}$ contribute to $\zeta$ like in the usual gravity. But the ghost modes provide the main impact to the comoving perturbation $B$. Since constant modes can be sewed exactly as in the Einstein gravity [mukhanov] we can sew remaining ghost modes through their contribution to $B$. The oscillating mode contribute to $B$ as $t^{-p / 2} e^{ \pm i m t}$. Connecting the solution for $B$ at the transition moment $t=1 / \mathrm{m}$ one can estimate for $t>1 / m$

$$
B=\frac{\zeta}{q} \sqrt{\frac{\epsilon}{3}}(m t)^{-p / 2}\left(\alpha_{+} e^{i m t}+\alpha_{-} e^{-i m t}\right),
$$

where $\alpha_{ \pm}$are expected to be constants of order unity.

\subsection{Coming back to the horizon}

In order to connect the superhorizon evolution of modes with the late subhorizon regime one can observe that the oscillating ghost modes resolve the equation on $W$ in both cases: $q / a<H$ and $q / a<H$. The only needed condition is $m>H$ which holds at late stages for all potentially observable modes. So the ghost modes come into the horizon remaining unchanged from which follows that the subhorizon evolution for constant mode is the same as in the usual gravity.

Since the presence of ghost breaks the Newtonian gauge we must find all observables using the comoving gauge. The difference from the usual gravity is only in the non-vanishing perturbation $B$ (32). In order to understand if this component provides any observable effect on the temperature anisotropy of $\mathrm{CMB}$ one need to consider its impact to the (integrated) Sachs-Wolfe effect. One may observe that the quantity $q B$ in the corresponding equations stands together with $\zeta$. So we can 

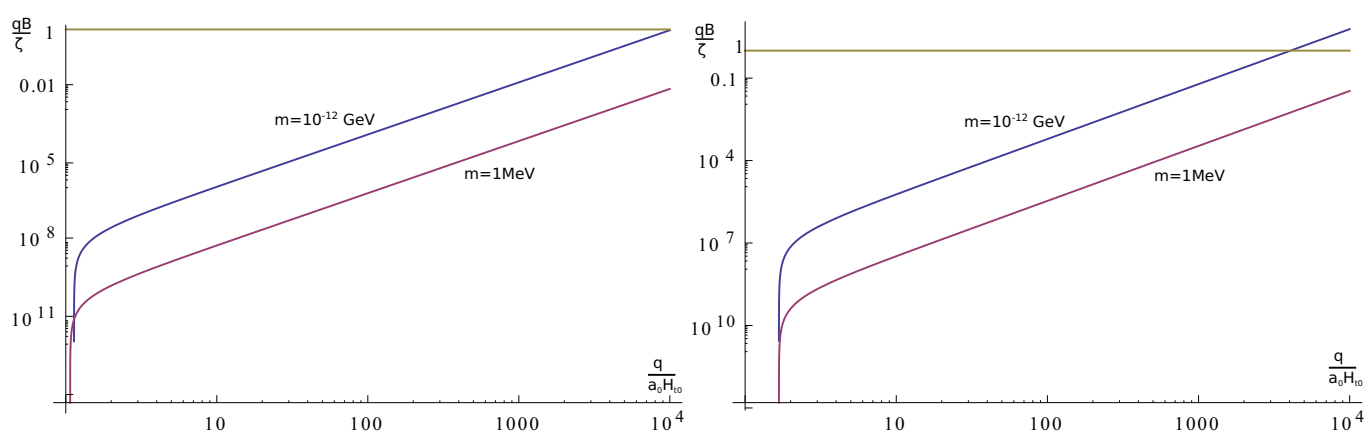

Figure 2. The left plot shows the value $q B / \zeta$ characterising the ghost contribution to the CMB anisotropy related to the present Universe. The right plot is for the moment of recombination. Here $q / a_{0}$ is the present physical momentum and $H_{t 0}$ is the present Hubble parameter. Directly observable modes correspond to $q / a_{0} H_{t 0}<1000$.

distinguish this case only if they are of the same order: $q B \sim \zeta$. The plots on Figure 2 shows the results related to the moment of recombination (right plot) and to the present moment (left plot). One can see that the deviations from the usual gravity may be expected only for short waves that we can not directly observe because they are in a strongly non-linear regime.

\section{Conclusions}

Summarising, we exploited the idea that extremely weak interacting ghost may be not so dangerous if we are considering the only effective description of the Universe. The instability arising from the small interaction between matter and ghost may be moved to the time much larger than the Universe age. This means that we can describe early Universe in terms of renormalizable and weakly interacting theory. We obtained the corrections to the observables, connected with scalar perturbations, due to extra ghost degrees of freedom to be negligible for the allowed ghost mass range.

In the considering theory, there are some processes with small but negative probabilities. The interpretation of such processes is an outstanding problem which we try to hide but actually it is impossible. Another problem for the non-unitary theory is that it remains unclear how to connect the quantum theory with bounded and positive energy, reached due to the negative norm prescription, to the classical field theory in which there is no way to avoid unbounded energy. This is an independent problem which is left beyond the scope of our consideration in this paper.

The author thank M. Ivanov, D. Gorbunov, A.A. Starobinsky, V.A. Rubakov, S.Sibiryakov for valuable discussions and critics. The work has been supported by Russian Science Foundation grant 14-12-01430.

\section{References}

[1] R. Kallosh, A. D. Linde, D. A. Linde and L. Susskind, Phys. Rev. D 52, 912 (1995) [hepth/9502069].

[2] F. Bezrukov, A. Magnin, M. Shaposhnikov and S. Sibiryakov, JHEP 1101, 016 (2011) [arXiv:1008.5157 [hep-ph]].

[3] K. S. Stelle, Gen. Rel. Grav. 9, 353 (1978).

[4] K. S. Stelle, Phys. Rev. D 16, 953 (1977). 
[5] A. Salvio and A. Strumia, JHEP 1406, 080 (2014) [arXiv:1403.4226 [hep-ph]].

[6] S. W. Hawking and T. Hertog, Phys. Rev. D 65, 103515 (2002) doi:10.1103/PhysRevD.65.103515 [hep-th/0107088].

[7] T. Clunan and M. Sasaki, Class. Quant. Grav. 27, 165014 (2010) [arXiv:0907.3868 [hep-th]].

[8] A.A. Starobinsky, Ya.B. Zeldovich, JETP Lett. 26, 252 (1977)

[9] A. Vilenkin, Phys. Rev. D 32, 2511 (1985).

[10] A. A. Geraci, S. J. Smullin, D. M. Weld, J. Chiaverini and A. Kapitulnik, Phys. Rev. D 78, 022002 (2008) [arXiv:0802.2350 [hep-ex]].

[11] G. Magnano, M. Ferraris and M. Francaviglia, Gen. Rel. Grav. 19, 465 (1987). doi:10.1007/BF00760651

[12] A. A. Starobinsky, Phys. Lett. B 91, 99 (1980).

[13] V. F. Mukhanov, H. A. Feldman and R. H. Brandenberger, Phys. Rept. 215, 203 (1992).

[14] N. Deruelle, M. Sasaki, Y. Sendouda and A. Youssef, JCAP 1103, 040 (2011) [arXiv:1012.5202 [gr-qc]]. 